\title{
Intake of energy-dense snack foods and drinks among Dutch children aged 7-12 years: how many, how much, when, where and which?
}

\author{
Dorus WM Gevers ${ }^{1, *}$, Stef PJ Kremers ${ }^{1}$, Nanne K de Vries ${ }^{1,2}$ and Patricia van Assema ${ }^{1}$ \\ 'Department of Health Promotion, NUTRIM School for Nutrition and Translational Research in Metabolism, \\ Maastricht University Medical Centre +, PO Box 616, 6200 MD Maastricht, The Netherlands: ${ }^{2}$ Department of Health \\ Promotion, CAPHRI School for Public Health and Primary Care, Maastricht University Medical Centre +, Maastricht, \\ The Netherlands
}

Submitted 21 August 2014: Final revision received 13 January 2015: Accepted 24 February 2015: First published online 8 April 2015

\begin{abstract}
Objective: To describe the energy-dense snack food (EDSF) and energy-dense drink (EDD) consumption of children in the Netherlands and investigate subgroup differences. The amounts consumed, eating occasions, places of consumption and consumed types are reported.

Design: Twenty-four hour dietary recall data were used to describe the EDSF and EDD consumption. Subgroup differences concerning these intakes were identified with ANCOVA.

Setting: Dutch National Food Consumption Survey 2007-2010.

Subjects: Children ( $n$ 860) aged 7-12 years.

Results: The mean number of EDSF events was 3.3 (SD 1.6) per day, yielding 1569.7 (SD 928.7) kJ. Average EDD consumption was 594.2 (SD 342.3) $\mathrm{ml} / \mathrm{d}$, yielding 737.2 ( $\mathrm{SD} 495.9$ ) $\mathrm{kJ}$. Over $90 \%$ of the children consumed more energy from non-core foods per day than recommended. Differences in EDSF and EDD consumption were found between several subgroups. Most importantly, we found higher intakes among older children and children with low educated mothers. Almost half of the EDSF events took place in the afternoon and at home. Cookies and sweets were consumed during half of the EDSF events. Almost one-third of the EDD were consumed in the afternoon. The majority of these drinks were consumed at home and most were soft drinks.

Conclusions: The results demonstrate that snack food and drink consumption is highly prevalent among Dutch children. Health promotion efforts addressing these behaviours are warranted and the present study could accelerate these initiatives. Focusing on children with low educated parents and on snacking at home after school offers the greatest potential to reduce snack food and drink intakes.
\end{abstract}

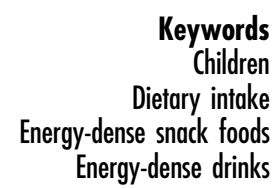

Chwords
Children

Dietary intake

Energy-dense drinks
The WHO and the FAO have identified high intake of energy-dense micronutrient-poor foods as a 'convincing causative factor of weight gain,(1). This category typically includes snack foods such as potato chips, chocolate and cookies. According to the WHO, a high intake of sugarsweetened soft drinks may also increase body weight and promote obesity ${ }^{(1)}$. Overweight in childhood is associated with short-term and long-term physical and psychological morbidity $^{(2,3)}$ and is likely to track into adulthood ${ }^{(4)}$. Independent of a high energy intake, high intakes of fat, sugar and salt from energy-dense snack foods (EDSF) or energy-dense drinks (EDD) may also result in harmful physical conditions (e.g. high blood pressure and high
LDL-cholesterol) and diseases (e.g. diabetes, CVD and dental caries $)^{(1)}$. There are indications that these dietary habits persist over time ${ }^{(5,6)}$.

Although there is no universally accepted definition of snack foods ${ }^{(7-9)}$, several nutrition institutes, including the Netherlands Nutrition Centre (NNC), distinguish core foods (e.g. fruit, vegetables and bread) from non-core foods (e.g. soft drinks, sauces and snacks) ${ }^{(10)}$. Due to the inconsistencies in definitions, some studies on snacking included the intake of all foods, including fruit and vegetables (e.g. reference 11), while others focused on noncore foods only (e.g. reference 12). The EDSF and EDD investigated in the present study belong to this non-core 
foods category. The NNC recommends a maximum daily consumption of 837-1255 kJ (200-300 kcal) from non-core foods, depending on age and $\operatorname{sex}^{(10)}$.

Policy makers and health promotion practitioners would benefit from information on the amounts of EDSF and EDD consumed and on risk groups, eating occasions, places of consumption and consumed types of food and drink. For several dietary behaviours, this information is available (e.g. reference 13), but few studies focus on EDSF and EDD and use representative samples of children and recent data. Macdiarmid et al. ${ }^{(12)}$ used national survey data collected in 2006 to investigate snacking patterns among Scottish children. They found a median number of $2.0 \mathrm{snacks} / \mathrm{d}^{(12)}$. Bell et al. ${ }^{(14)}$ reported that in 1995 the average proportion of energy from non-core foods and drinks in the daily energy intake of Australian children aged 5-12 years was over $40 \%$ for non-core foods and over $30 \%$ for non-core drinks. A recent European study reported that soft drink consumption among Dutch children aged 10-12 years was higher than that of their peers in six other European countries ${ }^{(15)}$. A recent report on the Dutch National Food Consumption Survey demonstrated that children aged 7-11 years consume almost $600 \mathrm{~g}$ of sugar-sweetened beverages daily ${ }^{(16)}$. Studies among various Dutch samples have shown means of nine to ten EDSF events per week ${ }^{(17,18)}$.

Children from low socio-economic backgrounds have been found to be at risk for high EDSF and EDD consumption $^{(19,20)}$. Differences in intakes between boys and girls and age groups have also been investigated ${ }^{(12,15,21)}$, but results are contradictory. Information concerning the occasions at which food is consumed has been reported in several studies. In particular, dietary patterns on weekdays have been compared with weekend days ${ }^{(22,23)}$ and the importance of after-school snacking has been highlighted $^{(24,25)}$. There is little information available on the places where EDSF and EDD are consumed, although home and school seem most common ${ }^{(26,27)}$. Some studies have reported the most commonly consumed types of EDSF and EDD, but their results are hard to compare due to differences of categorization ${ }^{(11,21)}$.

The current study aimed to describe the EDSF and EDD consumption of children aged 7-12 years in the Netherlands. Results can inform public health policies and interventions, and contribute to the international literature. The specific research aims of the study were to:

1. describe what quantities of EDSF and EDD children consume per day in terms of events, amounts and energy;

2. test if there are differences in EDSF and EDD consumption between sociodemographic subgroups and BMI subgroups; and

3. describe at what type of eating occasions and in what places children consume EDSF and EDD, and which types of EDSF and EDD are consumed.

\section{Methods}

Data were derived from the most recent Dutch National Food Consumption Survey, the DNFCS 2007-2010 ${ }^{(28)}$. That survey was commissioned by the Dutch Ministry of Health, Welfare and Sport and coordinated by the Dutch National Institute for Public Health and the Environment. Initially, 5502 members of a consumer panel aged 7-69 years were invited to participate and data collection took place through a market research agency (GfK Panel Services). In total, 3819 persons participated, 860 of whom were 7-12 years old.

\section{Data collection}

Data on food consumption were collected by trained dietitians by means of two $24 \mathrm{~h}$ dietary recalls using the EPIC-Soft software program ${ }^{(29)}$. Food products were matched with products from the Dutch Food Composition Database $(\mathrm{NEVO})^{(30)}$. Children were interviewed face-toface at home twice, in the presence of at least one parent or caregiver. There was an interval of about 4 weeks between the two interviews. Data collection was evenly distributed over the seasons and days of the week. In addition to the interviews, the children were given written questionnaires to assess demographic variables including age, sex, height and weight, employment status and country of birth of both parents, household income and household size. Information on respondents' place of residence (in terms of region and degree of urbanization) and the educational level of the head of the household was already known to the marketing research agency. A report on the full DNFCS 2007-2010, including the data collection method, is available ${ }^{(28)}$.

\section{Definitions of energy-dense snack foods and energy-dense drinks}

EDSF were defined using the type of eating occasion (during meals or between meals), as self-designated by the study participants, and using a food classification ${ }^{(14)}$ from the NNC. First, all foods classified as 'snacks' according to the NNC were identified as EDSF. The NNC defines snacks as foods that are meant for consumption between main meals. They include potato chips, nuts, savoury snacks, deep-fried snacks, cookies, pastry, sweets, chocolate, ice creams and certain types of dessert. Second, the type of eating occasion was taken into account for foods which often constitute or are part of a meal in the Netherlands, such as pizza, deep-fried snacks, including French fries and hamburgers, and ice creams. Hence, these types of food were defined as EDSF only when consumed between meals. Third, similar EDSF consumed within 60 min were considered to be part of the same snacking event and coded as such. Fourth, foods (both core foods and noncore foods) eaten in conjunction with an EDSF (e.g. hamburger with bun) were also considered to be part of the same snacking event and the total energy involved in this snack event was counted. 
EDD included in the present study were soft drinks and squash (drinks prepared from concentrated fruit syrups mixed with water), irrespective of the type of eating occasion. These drinks are included in the 'soft drinks' category of the non-core food group. Soft drinks with both sweeteners and added sugars were only included if the carbohydrate content was more than $2 \mathrm{~g}$ per $100 \mathrm{~g}^{(16)}$. Sugar-free beverages and fruit juices were not included in the definition of EDD.

\section{Data analysis}

BMI was calculated according to age- and sex-specific cutoff values ${ }^{(31,32)}$ and divided into underweight, normal weight and overweight (including obese). Based on definitions from Statistics Netherlands, the variables ethnicity, educational level of mother and education level of father were created ${ }^{(33,34)}$. Ethnicity consisted of three categories: native Dutch, Western immigrants and non-Western immigrants. Educational levels were defined as follows: low (primary school, lower vocational, low or intermediate general education), intermediate (intermediate vocational education and higher general education) and high (higher vocational education and university). Household income was assessed by nineteen categories of net income per month, which were reduced to three categories by splitting into tertiles (i.e. low, $<€ 1700$ net monthly; intermediate, $\geq € 1700$ to $<€ 2500$ net monthly, high, $\geq € 2500$ net monthly). Degree of urbanization was indicated by the number of addresses per square kilometre (i.e. low, $<1000$ addresses $/ \mathrm{km}^{2}$; intermediate, $\geq 1000$ and $<1500$ addresses $/ \mathrm{km}^{2}$; high, $\geq 1500$ addresses $/ \mathrm{km}^{2}$ ).

Descriptive analyses were used to describe the sample according to demographics and consumption in terms of the types of EDSF and numbers of EDSF events, types and amounts of EDD, energy intake from EDSF and EDD, the type of eating occasion and place of consumption. Descriptive statistics for the amount of, and energy from, EDSF and EDD were weighted for small deviations from the general Dutch population regarding sociodemographic characteristics (i.e. region where household is located, urbanization of household location and educational level of the household head), as well as for day of the week and the season of data collection. The weighing factor was derived by using census data from Statistics Netherlands (2008) ${ }^{(35)}$.

Differences in intakes (mean number of EDSF events per day, mean EDD intake in millilitres per day, and mean energy intake from EDSF and EDD events per day) according to age, sex, BMI, ethnicity, household income, degree of urbanization, educational level of the mother and father, employment status of the mother and father, and household size were identified using ANCOVA with age as a covariate while weighting for small deviations from the general Dutch population and moment of data collection. If the $F$ test showed a $P$ value $<0 \cdot 05$, Bonferroni post hoc tests were performed. Cohen's $d$ effect sizes for significantly different subgroups were calculated using group means and standard errors produced by the ANCOVA. Effect sizes were termed according to the classification of Lipsey ${ }^{(36)}$ : small $(\leq 0.32)$, medium $(0.33-0.55)$ and large $(>0.56)$. In order to determine the relative importance of the sociodemographic factors in explaining total EDSF and EDD intake and EDSF and EDD intake at home, weighted backward linear regression analyses were performed. To investigate determinants of intake at home, we created two new dependent variables called 'relative EDSF consumption at home' and 'relative EDD consumption at home', to rule out the influence of total consumption. These new variables were calculated by dividing the consumption at home by the total consumption. The intakes over two days were averaged for the analyses related to the amount of foods and drinks consumed. All analyses were conducted using the statistical software package IBM SPSS Statistics for Windows, version $20 \cdot 0$.

\section{Results}

The sample consisted of 860 children, with fewer respondents in the 9-10 years category than in the youngest and oldest age categories (Table 1). Boys and girls were evenly distributed, and $71 \%$ of the sample had a normal weight, while $20 \%$ were overweight and $10 \%$ were underweight. Overweight children and children of Dutch native ethnicity were somewhat over-represented, compared with the general Dutch population ${ }^{(35,37)}$. Compared with the general Dutch population, mothers and fathers with a high educational level were over-represented, while fathers and mothers with a low educational level were underrepresented $^{(35)}$. About $70 \%$ of the mothers and more than $90 \%$ of the fathers were employed. Almost half of the households consisted of four persons. The distribution of households across regions was comparable to that of the general Dutch population $^{(35)}$.

\section{Consumption of energy-dense snack foods and energy-dense drinks}

The mean number of EDSF events for the total sample was 3.3 (SD 1.6) per day (Table 2). The mean daily energy intake from EDSF was 1569.7 (sD 928.7) kJ for the total sample. The average amount of EDD consumed by the total sample was 594.2 (sD 342.3) $\mathrm{ml} / \mathrm{d}$. The mean energy intake from EDD for the total sample was $737 \cdot 2$ (SD $495 \cdot 9$ ) $\mathrm{kJ} / \mathrm{d}$.

The mean total energy intake from EDSF and EDD was 2306.8 (sD 1113.8 ) kJ/d (about $550 \mathrm{kcal} / \mathrm{d}$ ) and $91.1 \%$ of the children did not comply with the recommendation of the NNC (a maximum of 837-1255 kJ (200-300 kcal) from non-core foods per day, depending on age and $\left.\mathrm{sex}^{(5)}\right)$.

\section{Subgroup differences in energy-dense snack food and energy-dense drink consumption}

The 9- and 10-year-olds $(P<0.001)$ and the $11-$ and 12year-olds $(P=0.038)$ had significantly more events than 
Table 1 Characteristics of the study sample: children aged 7-12 years, Dutch National Food Consumption Survey 2007-2010

\begin{tabular}{|c|c|c|}
\hline & $n$ & Mean or \% \\
\hline \multicolumn{3}{|l|}{ Age (years) } \\
\hline Mean & 860 & 9.42 \\
\hline $\mathrm{SD}$ & & 1.75 \\
\hline \multicolumn{3}{|l|}{ Age $(\%)(n 860)$} \\
\hline 7 or 8 years & 304 & 35.3 \\
\hline 9 or 10 years & 265 & $30 \cdot 8$ \\
\hline 11 or 12 years & 291 & 33.8 \\
\hline \multicolumn{3}{|l|}{$\operatorname{Sex}(\%)(n 860)$} \\
\hline Male & 433 & $50 \cdot 3$ \\
\hline Female & 427 & 49.7 \\
\hline \multicolumn{3}{|l|}{ BMI group (\%) ( $n$ 859) } \\
\hline Underweight & 82 & 9.5 \\
\hline Normal weight & 608 & $70 \cdot 8$ \\
\hline Overweight and obese & 169 & $19 \cdot 7$ \\
\hline \multicolumn{3}{|l|}{ Ethnicity $(\%)(n 840)$} \\
\hline Native Dutch & 756 & 90 \\
\hline Western immigrant & 32 & 3.8 \\
\hline Non-Western immigrant & 52 & $6 \cdot 2$ \\
\hline \multicolumn{3}{|l|}{ Household income ( $n$ 860) } \\
\hline Low & 297 & 34.5 \\
\hline Intermediate & 268 & 31.2 \\
\hline High & 295 & 34.3 \\
\hline \multicolumn{3}{|l|}{ Degree of urbanization (\%) ( $n$ 860) } \\
\hline Low & 351 & $40 \cdot 8$ \\
\hline Intermediate & 150 & $17 \cdot 4$ \\
\hline High & 359 & 41.7 \\
\hline \multicolumn{3}{|l|}{ Educational level of mother ( $n$ 852) } \\
\hline Low & 290 & 34.0 \\
\hline Intermediate & 383 & $45 \cdot 0$ \\
\hline High & 179 & 21.0 \\
\hline \multicolumn{3}{|l|}{ Educational level of father $(n 758)$} \\
\hline Low & 221 & 29.2 \\
\hline Intermediate & 319 & $42 \cdot 1$ \\
\hline High & 218 & $28 \cdot 8$ \\
\hline \multicolumn{3}{|l|}{ Employment status of mother ( $n$ 849) } \\
\hline Employed & 614 & $72 \cdot 3$ \\
\hline Non-employed & 235 & $27 \cdot 7$ \\
\hline \multicolumn{3}{|l|}{ Employment status of father $(n 754)$} \\
\hline Employed & 719 & 95.4 \\
\hline Non-employed & 35 & 4.6 \\
\hline \multicolumn{3}{|l|}{ Household size ( $n$ 860) } \\
\hline 2 & 42 & 4.9 \\
\hline 3 & 163 & $19 \cdot 0$ \\
\hline 4 & 383 & 44.5 \\
\hline$\geq 5$ & 272 & 31.6 \\
\hline \multicolumn{3}{|l|}{ Region of household location ( $n 860$ ) } \\
\hline North & 96 & $11 \cdot 2$ \\
\hline East & 192 & $22 \cdot \overline{3}$ \\
\hline South & 207 & $24 \cdot 1$ \\
\hline Three largest cities in the West & 115 & 13.4 \\
\hline West (other) & 250 & $29 \cdot 1$ \\
\hline \multicolumn{3}{|l|}{ Season of interviews ( $n 860$ ) } \\
\hline Spring & 197 & $22 \cdot 9$ \\
\hline Summer & 216 & $25 \cdot 1$ \\
\hline Autumn & 222 & $25 \cdot 8$ \\
\hline Winter & 225 & $26 \cdot 2$ \\
\hline \multicolumn{3}{|c|}{ Day assessed by $24 \mathrm{~h}$ dietary recalls ( $n 860$ ) } \\
\hline Monday & 230 & 13.4 \\
\hline Tuesday & 297 & $17 \cdot 3$ \\
\hline Wednesday & 242 & $14 \cdot 1$ \\
\hline Thursday & 283 & $16 \cdot 5$ \\
\hline Friday & 246 & $14 \cdot 3$ \\
\hline Saturday & 202 & $11 \cdot 7$ \\
\hline Sunday & 220 & $12 \cdot 8$ \\
\hline
\end{tabular}

the 7- and 8-year-olds. Girls had significantly more events per day than boys $(P=0 \cdot 018)$. Children of native Dutch ethnicity had significantly more events per day than
non-Western immigrants $(P=0 \cdot 020)$ and children living in low-urbanization areas had more EDSF events than children living in highly urbanized areas $(P=0.005)$. Cohen's $d$ values of these differences were small to medium (ranged from $0 \cdot 16$ to $0 \cdot 40$; Table 2 ).

Children from the two older age categories had higher energy intake from EDSF than children from the youngest age category (both $P<0 \cdot 001$ ). Children of mothers with a low educational level consumed significantly more EDSF in $\mathrm{kJ} / \mathrm{d}$ than children of highly educated mothers $(P=0 \cdot 007)$. Children of fathers with low and intermediate educational levels consumed significantly more energy EDSF in $\mathrm{kJ} / \mathrm{d}$ than children of highly educated fathers $(P=0.012$ and $P=0.004$, respectively). Cohen's $d$ values of these differences were small to medium (ranged from 0.28 to 0.45 ; Table 2).

Children of mothers with a low educational level consumed significantly more EDD in $\mathrm{ml} / \mathrm{d}$ than children of mothers with a high educational level $(P=0.027$, Cohen's $d=0 \cdot 25$; Table 2).

More energy from EDD was consumed by children aged 11-12 years $(P=0.037)$ than by the youngest children. Children with a normal weight had higher energy intakes from EDD $(P=0.008)$ than underweight children. Children of mothers with a low and intermediate educational level ( $P=0.001$ and $P=0.041$, respectively), children of employed mothers $(P=0.034)$ and children from threeperson households $(P=0.024)$ consumed relatively more energy from EDD. Cohen's $d$ values of these differences were small to medium (ranged from $0 \cdot 16$ to $0 \cdot 35$; Table 2).

Age and the degree of urbanization were found to be significant correlates of the number of EDSF events after backward linear regression analyses (Table 3). With regard to energy intake from EDSF, the most important sociodemographic factors were age and the mother's educational level. Educational level of the mother was also a significant correlate of EDD consumption together with household size, both in terms of amount and energy. Energy intake from EDD was additionally explained by the child's BMI and mother's employment.

\section{Type of eating occasions, places of consumption and consumed types}

Almost half of the EDSF events took place in the afternoon and at home (Table 4). Cookies were consumed in onethird of all EDSF events and sweets in one-fifth of all EDSF events. Cookies included for instance Dutch raisin cookies, chocolate cookies and spiced cookies (i.e. Dutch windmill cookies). Sweets included mainly wine gums, Dutch liquorice, lollipops and boiled sweets. Almost onethird of the EDD were consumed in the afternoon. The majority of these drinks were consumed at home. The majority of EDD consumed were soft drinks, which included sodas such as coke and orangeade, fruit juice drinks, sports drinks and energy drinks.

Backward linear regression analyses showed that children living in large households (i.e. five persons) 
Table 2 Mean consumption of EDSF and EDD by sample characteristics among children aged 7-12 years, Dutch National Food Consumption Survey 2007-2010

\begin{tabular}{|c|c|c|c|c|c|c|c|c|}
\hline & \multicolumn{2}{|c|}{$\begin{array}{l}\text { EDSF events } \\
\text { (number/d) }\end{array}$} & \multicolumn{2}{|c|}{$\begin{array}{l}\text { Energy intake from EDSF } \\
(\mathrm{kJ} / \mathrm{d})\end{array}$} & \multicolumn{2}{|c|}{$\begin{array}{l}\text { EDD consumption } \\
(\mathrm{ml} / \mathrm{d})\end{array}$} & \multicolumn{2}{|c|}{$\begin{array}{l}\text { Energy intake from EDD } \\
\qquad(\mathrm{kJ} / \mathrm{d})\end{array}$} \\
\hline & Mean & SD & Mean & SD & Mean & SD & Mean & SD \\
\hline Total sample & $3 \cdot 3$ & $1 \cdot 6$ & $1569 \cdot 7$ & $928 \cdot 7$ & $594 \cdot 2$ & $342 \cdot 3$ & $737 \cdot 2$ & 495.9 \\
\hline Age & & & & & & & & \\
\hline (1) 7 or 8 years & 3.0 & 1.4 & $1320 \cdot 7$ & 709.6 & 568.4 & $315 \cdot 7$ & 674.2 & 456.9 \\
\hline (2) 9 or 10 years & 3.5 & 1.8 & $1668 \cdot 3$ & 957.9 & $630 \cdot 9$ & 323.5 & 763.4 & $450 \cdot 3$ \\
\hline (3) 11 or 12 years & 3.4 & 1.7 & 1729.9 & $1045 \cdot 0$ & 585.9 & 383.3 & 776.4 & 567.8 \\
\hline $\begin{array}{l}\text { Post hoc Bonferroni† } \\
\text { Cohen's dt }\end{array}$ & \multicolumn{2}{|c|}{$\begin{array}{c}2>1^{\star \star \star \star}, 3>1^{\star} \\
0.30,0.21\end{array}$} & \multicolumn{2}{|c|}{$\begin{array}{c}2>1^{\star \star \star \star \star}, 3>1^{\star \star \star \star} \\
0.38,0.45\end{array}$} & & & \multicolumn{2}{|c|}{$\begin{array}{l}3>1^{*} \\
0.21\end{array}$} \\
\hline Sex & & & & & & & & \\
\hline (1) Boys & $3 \cdot 2$ & 1.6 & $1572 \cdot 0$ & $957 \cdot 0$ & 610.9 & 355.5 & 747.9 & 519.9 \\
\hline (2) Girls & 3.4 & 1.7 & $1567 \cdot 2$ & 1411.6 & 576.4 & 327.8 & $725 \cdot 7$ & $470 \cdot 2$ \\
\hline $\begin{array}{l}\text { Post hoc Bonferroni } \\
\text { Cohen's } d\end{array}$ & \multicolumn{2}{|c|}{$\begin{array}{l}2>1^{*} \\
0.16\end{array}$} & \multicolumn{2}{|c|}{ NS } & \multirow{2}{*}{\multicolumn{2}{|c|}{ NS }} & \multirow{2}{*}{\multicolumn{2}{|c|}{ NS }} \\
\hline BMI & & & & & & & & \\
\hline (1) Underweight & 3.4 & 1.7 & $1532 \cdot 1$ & $822 \cdot 8$ & 527.8 & $298 \cdot 6$ & 588.6 & 398.4 \\
\hline (2) Normal weight & 3.3 & 1.7 & $1595 \cdot 1$ & 960.2 & 603.3 & 334.6 & 763.9 & $500 \cdot 3$ \\
\hline (3) Overweight or obese & $3 \cdot 2$ & 1.6 & $1501 \cdot 2$ & $872 \cdot 3$ & 595.2 & 389.3 & 714.8 & $516 \cdot 3$ \\
\hline $\begin{array}{l}\text { Post hoc Bonferroni } \\
\text { Cohen's } d\end{array}$ & \multicolumn{2}{|c|}{ NS } & \multicolumn{2}{|c|}{ NS } & \multicolumn{2}{|c|}{ NS } & \multicolumn{2}{|c|}{$\begin{array}{c}2>1^{\star *} \\
0.35\end{array}$} \\
\hline Ethnicity & & & & & & & & \\
\hline (1) Native Dutch & 3.4 & 1.6 & $1583 \cdot 8$ & $922 \cdot 2$ & 592.4 & $330 \cdot 0$ & 733.4 & 495.0 \\
\hline (2) Western immigrant & 3.4 & 1.7 & 1731.0 & $1056 \cdot 8$ & $616 \cdot 2$ & 358.5 & 828.4 & $524 \cdot 3$ \\
\hline (3) Non-Western immigrant & $2 \cdot 7$ & 1.8 & $1376 \cdot 2$ & 935.4 & 534.4 & 411.0 & 654.4 & 450.5 \\
\hline $\begin{array}{l}\text { Post hoc Bonferroni } \\
\text { Cohen's } d\end{array}$ & \multicolumn{2}{|c|}{$\begin{array}{c}1>3^{\star} \\
0.40\end{array}$} & \multicolumn{2}{|c|}{ NS } & & & & \\
\hline Household income & & & & & & & & \\
\hline (1) Low & $3 \cdot 3$ & 1.5 & $1642 \cdot 4$ & $915 \cdot 0$ & 621.5 & 359.7 & $752 \cdot 6$ & 479.3 \\
\hline (2) Intermediate & 3.4 & 1.9 & $1588 \cdot 3$ & 958.3 & 566.6 & $340 \cdot 1$ & $710 \cdot 4$ & 517.5 \\
\hline (3) High & $3 \cdot 2$ & 1.5 & $1476 \cdot 8$ & 914.6 & $592 \cdot 1$ & $326 \cdot 4$ & 746.4 & $495 \cdot 3$ \\
\hline Post hoc Bonferroni & & & & & & & & \\
\hline Degree of urbanization & & & & & & & & \\
\hline (1) Low & 3.5 & 1.7 & $1613 \cdot 7$ & $938 \cdot 8$ & $602 \cdot 1$ & 333.2 & 727.5 & $473 \cdot 2$ \\
\hline (2) Intermediate & $3 \cdot 2$ & 1.8 & $1502 \cdot 6$ & 1001.4 & $582 \cdot 2$ & 292.7 & 717.9 & 467.1 \\
\hline (3) High & $3 \cdot 1$ & 1.5 & 1561.6 & 887.0 & 592.8 & 373.3 & $755 \cdot 2$ & 531.1 \\
\hline $\begin{array}{l}\text { Post hoc Bonferroni } \\
\text { Cohen's } d\end{array}$ & $\begin{array}{r}1 \\
0\end{array}$ & & & & & & & \\
\hline Educational level of mother & & & & & & & & \\
\hline (1) Low & 3.4 & 1.7 & $1668 \cdot 3$ & 947.7 & $625 \cdot 1$ & 347.4 & $802 \cdot 0$ & $535 \cdot 6$ \\
\hline (2) Intermediate & 3.3 & 1.7 & 1593.6 & 946.8 & 600.9 & 341.3 & 744.0 & $490 \cdot 1$ \\
\hline (3) High & $3 \cdot 1$ & 1.4 & 1373.9 & 843.4 & $540 \cdot 8$ & 334.1 & $630 \cdot 2$ & 429.0 \\
\hline $\begin{array}{l}\text { Post hoc Bonferroni } \\
\text { Cohen's } d\end{array}$ & & & & & & & $\begin{array}{r}1> \\
0\end{array}$ & $\begin{array}{l}>3^{*} \\
22\end{array}$ \\
\hline Educational level of father & & & & & & & & \\
\hline (1) Low & 3.4 & $1 \cdot 7$ & $1630 \cdot 7$ & $930 \cdot 0$ & 607.9 & $330 \cdot 1$ & $760 \cdot 3$ & 485.5 \\
\hline (2) Intermediate & 3.4 & 1.6 & $1613 \cdot 1$ & 879.8 & 607.7 & 357.4 & $759 \cdot 3$ & 531.3 \\
\hline (3) High & $3 \cdot 1$ & 1.7 & $1380 \cdot 2$ & 911.4 & 553.8 & 308.9 & 662.4 & 444.3 \\
\hline $\begin{array}{l}\text { Post hoc Bonferroni } \\
\text { Cohen's } d\end{array}$ & & & $\begin{array}{r}1>3 \\
0\end{array}$ & & & & & \\
\hline Employment of mother & & & & & & & & \\
\hline (1) Employed & 3.3 & 1.6 & 1593.4 & 926.9 & 598.3 & 337.0 & 761.9 & $512 \cdot 7$ \\
\hline (2) Non-employed & $3 \cdot 3$ & $1 \cdot 7$ & $1518 \cdot 2$ & 941.9 & 593.4 & $358 \cdot 1$ & 684.0 & $450 \cdot 7$ \\
\hline $\begin{array}{l}\text { Post hoc Bonferroni } \\
\text { Cohen's } d\end{array}$ & & & & & & & 0.16 & \\
\hline Employment of father & & & & & & & & \\
\hline (1) Employed & 3.4 & $1 \cdot 7$ & $1558 \cdot 6$ & 906.9 & 594.5 & 337.8 & 731.6 & $494 \cdot 3$ \\
\hline (2) Non-employed & $2 \cdot 8$ & 1.3 & 1462.9 & 891.3 & $622 \cdot 2$ & 359.9 & 801.7 & 585.4 \\
\hline Post hoc Bonferroni & & & & & & & & \\
\hline Household size & & & & & & & & \\
\hline (1) 2 & $3 \cdot 2$ & 1.8 & $1559 \cdot 4$ & $1475 \cdot 3$ & 521.4 & $370 \cdot 6$ & 678.0 & 437.5 \\
\hline (2) 3 & $3 \cdot 1$ & 1.4 & $1692 \cdot 5$ & $987 \cdot 2$ & 627.9 & $340 \cdot 2$ & $805 \cdot 1$ & 489.9 \\
\hline (3) 4 & $3 \cdot 3$ & 1.6 & $1540 \cdot 1$ & $908 \cdot 7$ & 614.3 & 354.1 & 765.1 & 532.7 \\
\hline (4) $\geq 5$ & 3.4 & 1.8 & $1539 \cdot 3$ & $927 \cdot 0$ & 557.2 & $320 \cdot 6$ & $665 \cdot 7$ & 448.6 \\
\hline $\begin{array}{l}\text { Post hoc Bonferroni } \\
\text { Cohen's } d\end{array}$ & & & & & & & & \\
\hline
\end{tabular}

EDSF, energy-dense snack food; EDD, energy-dense drink.

Analyses were weighted for small deviations in sociodemographic characteristics, day of the week and season of data collection; age was included as a covariate. ${ }^{\star} P<0.05,{ }^{\star \star} P<0.01,{ }^{\star \star \star} P<0.005,{ }^{\star \star \star \star} P<0.001$.

TANCOVA without age as a covariate.

¥Cohen's $d$ values were calculated from subgroup means and standard errors produced by ANCOVA (with age as a covariate): Cohen's $d=\left(\bar{x}_{a}-\bar{x}_{b}\right) / \sqrt{\frac{\left[\left(n_{a}-1\right)\left(S E_{a} \sqrt{n_{a}}\right)^{2}+\left(n_{b}-1\right)\left(S E_{b} \sqrt{n_{b}}\right)^{2}\right]}{\left(n_{a}+n_{b}\right)}}$. 
Table 3 Relative importance of child background characteristics in explaining EDSF and EDD consumption, children aged 7-12 years, Dutch National Food Consumption Survey 2007-2010

\begin{tabular}{|c|c|c|c|c|c|c|c|c|}
\hline & \multicolumn{2}{|c|}{$\begin{array}{c}\text { EDSF events } \\
\text { (number/d, } n \text { 860) }\end{array}$} & \multicolumn{2}{|c|}{$\begin{array}{l}\text { Energy intake from EDSF } \\
(\mathrm{kJ} / \mathrm{d}, n \text { 852) }\end{array}$} & \multicolumn{2}{|c|}{$\begin{array}{l}\text { EDD consumption } \\
(\mathrm{ml} / \mathrm{d}, n 852)\end{array}$} & \multicolumn{2}{|c|}{$\begin{array}{l}\text { Energy intake from EDD } \\
(\mathrm{kJ} / \mathrm{d}, n \text { 847) }\end{array}$} \\
\hline & $\beta$ & $P$ value & $\beta$ & $P$ value & $\beta$ & $P$ value & $\beta$ & $P$ value \\
\hline Age & 0.08 & 0.03 & 0.17 & $<0.01$ & & & & \\
\hline \multicolumn{9}{|l|}{$\begin{array}{l}\text { Sex } \\
\text { Boys (reference) } \\
\text { Girls }\end{array}$} \\
\hline \multicolumn{9}{|l|}{ BMI } \\
\hline $\begin{array}{l}\text { Underweight } \\
\text { Normal weight (reference) }\end{array}$ & & & & & & & $-0 \cdot 10$ & $<0.01$ \\
\hline Overweight & & & & & & & -0.05 & 0.16 \\
\hline \multicolumn{9}{|l|}{ Ethnicity } \\
\hline \multicolumn{9}{|l|}{ Native Dutch (reference) } \\
\hline \multicolumn{9}{|l|}{ Western immigrant } \\
\hline \multicolumn{9}{|l|}{ Non-Western immigrant } \\
\hline \multicolumn{9}{|l|}{ Household income } \\
\hline \multicolumn{9}{|l|}{ Low (reference) } \\
\hline \multicolumn{9}{|l|}{ Intermediate } \\
\hline \multicolumn{9}{|l|}{ High } \\
\hline \multicolumn{9}{|l|}{ Degree of urbanization } \\
\hline \multicolumn{9}{|l|}{ Low (reference) } \\
\hline Intermediate & -0.07 & 0.05 & & & & & & \\
\hline High & -0.11 & $<0.01$ & & & & & & \\
\hline \multicolumn{9}{|l|}{ Educational level mother } \\
\hline \multicolumn{9}{|l|}{ Low (reference) } \\
\hline Intermediate & & & 0.03 & 0.39 & -0.04 & 0.36 & -0.08 & 0.04 \\
\hline High & & & $-0 \cdot 12$ & $<0.01$ & $-0 \cdot 10$ & $<0.01$ & -0.17 & $<0.01$ \\
\hline \multicolumn{9}{|l|}{ Educational level father } \\
\hline \multicolumn{9}{|l|}{ Low (reference) } \\
\hline \multicolumn{9}{|l|}{ Intermediate } \\
\hline \multicolumn{9}{|l|}{ High } \\
\hline \multirow{2}{*}{\multicolumn{9}{|c|}{$\begin{array}{l}\text { Employment of mother } \\
\text { Employed (reference) }\end{array}$}} \\
\hline & & & & & & & & \\
\hline Non-employed & & & & & & & -0.09 & 0.01 \\
\hline \multicolumn{9}{|l|}{ Employment of father } \\
\hline \multicolumn{9}{|l|}{ Employed (reference) } \\
\hline Non-employed & & & & & & & & \\
\hline Household size & & & & & & & & \\
\hline 2 & & & & & -0.03 & 0.44 & $<0.01$ & 0.99 \\
\hline 3 & & & & & 0.07 & 0.07 & 0.09 & 0.03 \\
\hline 4 & & & & & 0.08 & 0.05 & 0.09 & 0.02 \\
\hline$\geq 5$ (reference) & & & & & & & & \\
\hline$R^{2}$ & 0.02 & & 0.04 & & 0.02 & & 0.05 & \\
\hline
\end{tabular}

EDSF, energy-dense snack food; EDD, energy-dense drink.

Analyses were weighted for small deviations in sociodemographic characteristics, day of the week and season of data collection.

consumed a greater proportion of EDSF at home than children living in small households (i.e. two persons; $\left.\beta=-0.11, \quad P=0.002, \quad R^{2}=0.01\right)$. These analyses also showed that children of unemployed mothers consumed a greater proportion of EDD at home than children with employed mothers $\left(\beta=0.07, P=0.03, R^{2}=0.01\right)$ and that children living in low urbanized areas consumed a greater proportion of EDD at home than children living in high urbanized areas $\left(\beta=0.02, P=0.03, R^{2}=0.01\right)$.

\section{Discussion}

The current study examined the consumption of EDSF and EDD among Dutch children in terms of the amounts consumed, type of eating occasions, places of consumption and consumed types. The results demonstrated that, on average, Dutch children aged between 7 and 12 years consumed more than three EDSF per day, yielding over $1500 \mathrm{~kJ}$. The number of snacking events in the current study was higher than that found among Scottish children ${ }^{(12)}$ and in Dutch studies investigating specific sub-populations $^{(17,18)}$. On average, the children consumed almost $600 \mathrm{ml}$ of EDD daily, which is comparable to the outcomes of the European study by Brug et $a l .{ }^{(15)}$. In the current study, the average energy intake from EDD was more than $730 \mathrm{~kJ} / \mathrm{d}$. There are no similar studies available with which to compare our results concerning the energy intake from EDSF and EDD. Nevertheless, these results indicate that efforts to promote healthy dietary behaviours in Dutch children are justified and necessary, as their energy intakes from EDSF and 
Table 4 Proportions of EDSF and EDD consumed according to eating occasion, place and type, children aged 7-12 years, Dutch National Food Consumption Survey 2007-2010

\begin{tabular}{|c|c|c|}
\hline & $\begin{array}{l}\text { Proportion of total } \\
\text { EDSF } \\
\text { consumption (\%) }\end{array}$ & $\begin{array}{c}\text { Proportion of total } \\
\text { EDD } \\
\text { consumption (\%) }\end{array}$ \\
\hline \multicolumn{3}{|l|}{ Type of eating occasion } \\
\hline Before breakfast & 0.5 & 0.6 \\
\hline During breakfast & 1.6 & 5.7 \\
\hline $\begin{array}{l}\text { During the morning } \\
\text { (between breakfast and } \\
\text { lunch) }\end{array}$ & 23.0 & $20 \cdot 5$ \\
\hline During lunch & 4.9 & $10 \cdot 3$ \\
\hline $\begin{array}{l}\text { During the afternoon } \\
\text { (between lunch and } \\
\text { dinner) }\end{array}$ & $45 \cdot 3$ & 31.9 \\
\hline During dinner & 1.7 & 11.3 \\
\hline $\begin{array}{l}\text { During the evening/at } \\
\text { night }\end{array}$ & $23 \cdot 0$ & 19.7 \\
\hline \multicolumn{3}{|l|}{ Weekdays $v$. weekend days $\dagger$} \\
\hline Weekdays & 47.4 & $49 \cdot 1$ \\
\hline Weekend days & $52 \cdot 6$ & $50 \cdot 9$ \\
\hline \multicolumn{3}{|l|}{ Place of consumption } \\
\hline At home & $49 \cdot 3$ & $58 \cdot 0$ \\
\hline At school & $17 \cdot 1$ & $17 \cdot 7$ \\
\hline At a friend's home & $15 \cdot 2$ & $12 \cdot 4$ \\
\hline $\begin{array}{l}\text { Other (i.e. street, sports } \\
\text { centre, car/boat/train/ } \\
\text { airplane, café/restaurant, } \\
<5 \% \text { per group) }\end{array}$ & $18 \cdot 4$ & 11.9 \\
\hline \multicolumn{3}{|l|}{ Type of EDSF } \\
\hline $\begin{array}{l}\text { Cookies (e.g. Dutch } \\
\text { raisin cookies, } \\
\text { chocolate cookies) }\end{array}$ & $30 \cdot 9$ & \\
\hline $\begin{array}{l}\text { Sweets (e.g. wine gums, } \\
\text { Dutch liquorice) }\end{array}$ & $21 \cdot 2$ & \\
\hline Potato chips & 8.5 & \\
\hline $\begin{array}{l}\text { Chocolate (excluding } \\
\text { candy bars) }\end{array}$ & $7 \cdot 3$ & \\
\hline Pastry & $6 \cdot 0$ & \\
\hline Chewing gum and mints & $6 \cdot 0$ & \\
\hline Ice cream & 4.5 & \\
\hline $\begin{array}{l}\text { Savoury snacks (e.g. } \\
\text { pretzel sticks) and nuts } \\
\text { (e.g. coated peanuts) }\end{array}$ & $4 \cdot 3$ & \\
\hline Ginger cake & 2.5 & \\
\hline Candy bars & 2.0 & \\
\hline $\begin{array}{l}\text { Deep-fried snacks (e.g. } \\
\text { French fries) }\end{array}$ & $1 \cdot 8$ & \\
\hline Other & $5 \cdot 0$ & \\
\hline \multicolumn{3}{|l|}{ Type of EDD } \\
\hline $\begin{array}{l}\text { Soft drink (e.g. sodas, } \\
\text { sports drinks, energy } \\
\text { drinks, fruit juice drinks) }\end{array}$ & & $60 \cdot 7$ \\
\hline $\begin{array}{l}\text { Squash (concentrated } \\
\text { fruit syrups mixed with } \\
\text { water) }\end{array}$ & & $39 \cdot 3$ \\
\hline
\end{tabular}

EDSF, energy-dense snack food; EDD, energy-dense drink. †Percentages based on cases with one recall day during the week and one recall day during the weekend ( $n$ 294).

EDD exceed the recommendations from the $\mathrm{NNC}^{(10)}$. The total mean intake from these foods and drinks was over $2300 \mathrm{~kJ} / \mathrm{d}$ (about $550 \mathrm{kcal} / \mathrm{d}$ ) and as many as $90 \%$ of the children exceeded the recommendation (a maximum of $837-1255 \mathrm{~kJ}$ (200-300 kcal) from non-core foods per day, depending on age and $\operatorname{sex}^{(10)}$ ).
Older children had higher intakes of EDSF and EDD than younger children. Older children are generally likely to have higher total daily energy intakes than younger children, while the proportion of snack foods to the total daily energy intake may increase with age as well ${ }^{(21)}$. Intervening just before the age of 9 years might be the most effective option to reduce EDSF and EDD consumption, as we found a steep rise in these behaviours from this age onwards, indicated by medium sized effects ${ }^{(36)}$. Considering all factors included in the present study, age appeared to be one of the most important predictors of EDSF consumption. Girls, children of native Dutch ethnicity and children living in low-urbanization areas had relatively more EDSF events. Macdiarmid et al. ${ }^{(12)}$ did not find differences in the number of snacking events between Scottish boys and girls. Future studies should examine sex differences in the portion sizes and types of EDSF and EDD consumed. We found no other studies that reported results concerning urbanization, and we have no a clear explanation for the difference we observed. The finding that non-Western immigrants had fewer EDSF events may be explained by the underrepresentation of this group in the sample, as non-Dutch speaking persons were excluded from the study. Brug et $a l .{ }^{(38)}$ found no differences in soft drink consumption between children of non-native ethnicity and native ethnicity in the Netherlands, which is in agreement with the current findings. The finding that overweight and obese children did not differ from normal-weight or underweight children in their intakes of EDSF and EDD may be caused by under-reporting ${ }^{(39)}$, dieting behaviour ${ }^{(40)}$ or low levels of energy expenditure ${ }^{(41)}$ by overweight children. Less favourable outcomes regarding EDSF and EDD consumption were found among children with lower educated parents. The mother's educational level was found to be the most important sociodemographic predictor of a child's EDD intake. Other studies have amply documented higher intakes of EDSF among children of low socioeconomic status compared with children of high socioeconomic status ${ }^{(15,19)}$. Hence, interventions to promote healthy eating should prioritize children with lower educated parents, taking into consideration the mechanisms that make these children consume more EDSF and EDD, such as food parenting practices, parental consumption and food availability at home $e^{(20,42,43)}$. Children with mothers in employment had higher energy intakes from EDD than children with non-employed mothers. Earlier studies have also acknowledged the role of mothers' working hours in children's diets and found a positive association between maternal working hours and intakes of soft drinks and fast food ${ }^{(44)}$. The most plausible explanation for this finding is lack of time, as working mothers may have less time to prepare healthy meals ${ }^{(44)}$ or monitor their child's dietary intake ${ }^{(45)}$. Children living in households consisting of three individuals had a higher energy intake from EDD than children living in the largest 
households. Ayala et al. ${ }^{(46)}$ did not find a relationship between household size and soft drink consumption, but found a positive relationship between household size and snack food intake. Neither their study nor ours collected data on family composition (e.g. one-parent families), so it is possible that relationships between household size and dietary intakes interact with other factors.

Half of all EDSF and a third of the EDD events occurred in the afternoon, most often at home. The importance of after-school snacking has also been found among children in other countries ${ }^{(24,25)}$. Intervention objectives should therefore not be limited to reducing EDSF intake at schools. We found that children living in large households, children of unemployed mothers and children living in low urbanized areas had a relatively high proportional intake of EDSF or EDD at home. Probably, these children and their mothers spend more time at home. Still, research should pay more attention to factors underlying after-school snacking. Popular types of EDSF were cookies and sweets. These foods often contain nutrients that may have harmful effects, such as sugar and fat. Most EDD that were consumed consisted of soft drinks. Few studies have distinguished between snack food consumption during meals and consumption between meals. In the present study, there was a modest contribution of energy from snack foods consumed during meals and a moderate contribution of energy from EDD consumed during meals to the total consumption of these foods and drinks. Nevertheless, both could have unfavourable effects, as those foods and drinks may replace nutritious foods served at meals. The current study did not find major differences in intakes of EDSF and EDD between weekdays and weekend days, although several studies have observed such differences ${ }^{(12,22,47,48)}$. In the current sample, differences between weekdays and weekend days might be present in the energy derived from these foods or drinks, or the types consumed. Additionally, differences in these behaviours could be captured in day-to-day variations, which might be explored in future studies.

It is likely that dietary patterns, and particularly the type of eating occasion and place of EDSF and EDD consumption, vary between countries due to differences in school hours and policies concerning school meals. In the Netherlands, primary schools usually start around 08:30 hours and end around 15:15 hours, with a short break in the morning and a one-hour break at lunchtime. Children may go home for lunch or may stay at school. The results of the present study should be interpreted in the light of this context.

The current sample is representative of the Dutch population in many characteristics. However, overweight children, children of native Dutch ethnicity and children with highly educated parents were somewhat overrepresented. The method of data collection in the current study carries a certain risk of memory and recording biases $^{(49)}$. However, these biases were minimized by using trained dietitians and a computer-based recording system.
The data that were used probably do not fully represent habitual intake, as the habitual intake may deviate from reported recall days ${ }^{(50)}$. However, reported intakes were based on two recall days, approaching habitual intake more accurately than a single $24 \mathrm{~h}_{\text {recall }}{ }^{(51)}$.

\section{Conclusions}

The data from the present study demonstrate that snack food and drink consumption is highly prevalent among Dutch children. Health promotion efforts addressing these behaviours are warranted and can benefit from our findings regarding the specific groups at risk, eating occasions and places involving higher risks, and the types of foods and drinks that are frequently consumed. Particularly, focusing on children with low educated parents and on snacking at home after school offers the greatest potential to reduce snack food and drink intake.

\section{Acknowledgements}

Acknowledgements: Data analysed were from FCS2010_Core_20111125; part of the Dutch National Food Consumption Survey, performed by the National Institute for Public Health and the Environment (RIVM), Bilthoven. Financial support: This research received no specific grant from any funding agency in the public, commercial or notfor-profit sectors. Conflict of interest: None. Authorship: All authors contributed to the formulation of the research questions and the design of the study. D.W.M.G. performed the statistical analysis and drafted the first version of the manuscript. All authors made substantial contributions in improving the manuscript and read and approved the final manuscript. Ethics of buman subject participation: The DNFCS 2007-2010 was conducted according to the guidelines laid down in the Declaration of Helsinki.

\section{References}

1. World Health Organization (2003) Diet, Nutrition and the Prevention of Chronic Diseases. Joint WHO/FAO Expert Consultation. WHO Technical Report Series no. 916. Geneva: WHO.

2. Reilly JJ \& Kelly J (2011) Long-term impact of overweight and obesity in childhood and adolescence on morbidity and premature mortality in adulthood: systematic review. Int J Obes (Lond) 35, 891-898.

3. Reilly JJ, Methven E, McDowell ZC et al. (2003) Health consequences of obesity. Arch Dis Child 88, 748-752.

4. Singh AS, Mulder C, Twisk JW et al. (2008) Tracking of childhood overweight into adulthood: a systematic review of the literature. Obes Rev $9,474-488$.

5. Totland TH, Gebremariam MK, Lien N et al. (2013) Does tracking of dietary behaviours differ by parental education in children during the transition into adolescence? Public Health Nutr 16, 673-682. 
6. Pearson N, Salmon J, Campbell K et al. (2011) Tracking of children's body-mass index, television viewing and dietary intake over five-years. Prev Med 53, 268-270.

7. Gregori D, Foltran F, Ghidina M et al. (2011) Understanding the influence of the snack definition on the association between snacking and obesity: a review. Int J Food Sci Nutr 62, 270-275.

8. Gregori D \& Maffeis C (2007) Snacking and obesity: urgency of a definition to explore such a relationship. J Am Diet Assoc 107, 562-563.

9. Johnson GH \& Anderson GH (2010) Snacking definitions: impact on interpretation of the literature and dietary recommendations. Crit Rev Food Sci Nutr 50, 848-871.

10. Netherlands Nutrition Centre (2011) Richtlijnen voedselkeuze (Guidelines for Food Choices). The Hague: Netherlands Nutrition Centre; available at http://www.voedingscentrum.nl/ Assets/Uploads/voedingscentrum/Documents/Professionals/ Voedselvoorlichting/01_Richtlijnen\%20voedselkeuze\%20 Voedingscentrum.pdf

11. Kerr MA, Rennie KL, McCaffrey TA et al. (2009) Snacking patterns among adolescents: a comparison of type, frequency and portion size between Britain in 1997 and Northern Ireland in 2005. Br J Nutr 101, 122-131.

12. Macdiarmid J, Loe J, Craig LC et al. (2009) Meal and snacking patterns of school-aged children in Scotland. Eur J Clin Nutr 63, 1297-1304.

13. Piernas C, Barquera S \& Popkin BM (2014) Current patterns of water and beverage consumption among Mexican children and adolescents aged $1-18$ years: analysis of the Mexican National Health and Nutrition Survey 2012. Public Health Nutr 17, 2166-2175.

14. Bell AC, Kremer PJ, Magarey AM et al. (2005) Contribution of 'noncore' foods and beverages to the energy intake and weight status of Australian children. Eur J Clin Nutr 59, 639-645.

15. Brug J, van Stralen MM, te Velde SJ et al. (2012) Differences in weight status and energy-balance related behaviors among schoolchildren across Europe: the ENERGY-project. PLoS One 7, e34742.

16. Buurma-Rethans E, Fransen H \& van Rossum C (2012) Consumptie van dranken met toegevoegd suiker door kinderen van de basisschoolleeftijd (Consumption of beverages with added sugars among primary school children). http://www.rivm.nl/ Documenten_en_publicaties/Algemeen_Actueel/Uitgaven/Lee fstijl_Voeding/VCP/Consumptie_van_dranken_met_toegevo egd_suiker_door_kinderen_van_de_basisschoolleeftijd (accessed July 2014).

17. Van Strien T, van Niekerk R \& Ouwens MA (2009) Perceived parental food controlling practices are related to obesogenic or leptogenic child life style behaviors. Appetite 53, 151-154.

18. Rodenburg G, Kremers SP, Oenema A et al. (2014) Associations of parental feeding styles with child snacking behaviour and weight in the context of general parenting. Public Health Nutr 17, 960-969.

19. Craig LCA, McNeill G, Macdiarmid JI et al. (2010) Dietary patterns of school-age children in Scotland: association with socio-economic indicators, physical activity and obesity. Br J Nutr 103, 319-334.

20. Totland TH, Lien N, Bergh IH et al. (2013) The relationship between parental education and adolescents' soft drink intake from the age of 11-13 years, and possible mediating effects of availability and accessibility. Br J Nutr 110, 926-933.

21. Rangan AM, Randall D, Hector DJ et al. (2008) Consumption of 'extra' foods by Australian children: types, quantities and contribution to energy and nutrient intakes. Eur J Clin Nutr 62, 356-364.

22. Rothausen BW, Matthiessen J, Andersen LF et al. (2013) Dietary patterns on weekdays and weekend days in 4-14year-old Danish children. Br J Nutr 109, 1704-1713.
23. Rockell JE, Parnell WR, Wilson NC et al. (2011) Nutrients and foods consumed by New Zealand children on schooldays and non-schooldays. Public Health Nutr 14, 203-208.

24. Rockell JE, Skidmore PML, Parnell WR et al. (2011) What children eat during afternoons and evenings: is it important? Public Health Nutr 14, 557-562.

25. Gilbert J-A, Miller D, Olson S \& St Pierre S (2012) Afterschool snack intake among Canadian children and adolescents. Can J Public Health 103, e448-e452.

26. Briefel RR, Wilson A \& Gleason PM (2009) Consumption of low-nutrient, energy-dense foods and beverages at school, home, and other locations among school lunch participants and nonparticipants. J Am Diet Assoc 109, 2 Suppl., S79-S90,

27. Kerr MA, McCrorie TA, Rennie KL et al. (2010) Snacking patterns according to location among Northern Ireland children. Int J Pediatr Obes 5, 243-249.

28. Van Rossum CTM, Fransen HP, Verkaik-Kloosterman J et al. (2011) Dutch National Food Consumption Survey 2007-2010. Diet of Children and Adults Aged 7 to 69 Years. Bilthoven: RIVM; available at http://www.rivm.nl/Documenten_en_ publicaties/Wetenschappelijk/Rapporten/2011/oktober/Dutch_ National_Food_Consumption_Survey_2007_2010_Diet_of_ children_and_adults_aged_7_to_69_years

29. Slimani N, Ferrari P, Ocke M et al. (2000) Standardization of the 24-hour diet recall calibration method used in the European Prospective Investigation into Cancer and Nutrition (EPIC): general concepts and preliminary results. Eur J Clin Nutr 54, 900-917.

30. National Institute for Public Health and the Environment (2011) NEVO-tabel. Nederlands Voedingsstoffenbestand 2011 (Dutch Food Composition Database 2011). Den Haag: RIVM.

31. Van Buuren S (2004) Afkapwaarden van de Bodymass index voor de bepaling van ondergewicht bij Nederlandse kinderen (Body-mass index cut-off values for underweight in Dutch children). Ned Tijdsch Geneesk 148, 1967-1972.

32. Hirasing RA, Fredriks AM, van Buuren S et al. (2001) Toegenomen prevalentie van overgewicht en obesitas bij Nederlandse kinderen en signalering daarvan aan de hand van internationale normen en nieuwe referentiediagrammen (Increased prevalence of overweight and obesity in Dutch children, and the detection of overweight and obesity using international criteria and new reference diagrams). Ned Tijdschr Geneeskd 145, 1303-1308.

33. Keij I (2000) Standaarddefinitie allochtonen. Index 10, 24-25.

34. Statistics Netherlands (2015) International Standard Classification of Education. http://www.cbs.nl/nl-NL/menu/ methoden/classificaties/overzicht/isced/default.htm (accessed March 2015).

35. Statistics Netherlands (n.d.) Statline databse. http://statline. cbs.nl/ (accessed July 2014).

36. Lipsey M (1990) Design Sensitivity: Statistical Power for Experimental Research. Newbury Park, CA: SAGE Publications.

37. Schonbeck Y, Talma H, van Dommelen P et al. (2011) Increase in prevalence of overweight in Dutch children and adolescents: a comparison of nationwide growth studies in 1980, 1997 and 2009. PLoS One 6, e27608.

38. Brug J, van Stralen MM, Chinapaw MJ et al. (2012) Differences in weight status and energy-balance related behaviours according to ethnic background among adolescents in seven countries in Europe: the ENERGY-project. Pediatr Obes 7, 399-411.

39. Fisher JO, Johnson RK, Lindquist C et al. (2000) Influence of body composition on the accuracy of reported energy intake in children. Obes Res $\mathbf{8}, 597-603$.

40. Hill AJ \& Pallin V (1998) Dieting awareness and low selfworth: related issues in 8-year-old girls. Int J Eat Disord 24, 405-413. 
41. Rennie KL, Johnson L \& Jebb SA (2005) Behavioural determinants of obesity. Best Pract Res Clin Endocrinol Metab 19, 343-358.

42. De Coen V, Vansteelandt S, Maes L et al. (2012) Parental socioeconomic status and soft drink consumption of the child. The mediating proportion of parenting practices. Appetite 59, 76-80.

43. Zarnowiecki DM, Dollman J \& Parletta N (2014) Associations between predictors of children's dietary intake and socioeconomic position: a systematic review of the literature. Obes Rev 15, 375-391.

44. Datar A, Nicosia N \& Shier V (2014) Maternal work and children's diet, activity, and obesity. Soc Sci Med 107, 196-204.

45. Gubbels JS, Kremers SP, Stafleu A et al. (2011) Association between parenting practices and children's dietary intake, activity behavior and development of body mass index: the KOALA Birth Cohort Study. Int J Behav Nutr Phys Act 8, 18 .
46. Ayala GX, Baquero B, Arredondo EM et al. (2007) Association between family variables and Mexican American children's dietary behaviors. J Nutr Educ Behav 39, 62-69.

47. Bjelland M, Lien N, Grydeland M et al. (2011) Intakes and perceived home availability of sugar-sweetened beverages, fruit and vegetables as reported by mothers, fathers and adolescents in the HEIA (HEalth In Adolescents) study. Public Health Nutr 14, 2156-2165.

48. Hart CN, Raynor HA, Osterholt KM et al. (2011) Eating and activity habits of overweight children on weekdays and weekends. Int J Pediatr Obes 6, 467-472.

49. Willett WC (1998) Nutritional Epidemiology, 2nd ed. New York: Oxford University Press.

50. Mackerras D \& Rutishauser I (2005) 24-hour national dietary survey data: how do we interpret them most effectively? Public Health Nutr 8, 657-665.

51. Guenther PM, Dodd KW, Reedy J et al. (2006) Most Americans eat much less than recommended amounts of fruits and vegetables. J Am Diet Assoc 106, 1371-1379. 